\title{
DEFORMATION MAPPING FOR COAL MINING USING TIME-SERIES INSAR COMBINING PERSISTENT SCATTERER AND DISTRIBUTED SCATTERER IN HUAIN CITY, CHINA
}

\author{
Zhengjia Zhang $^{(1),(2)}$, Yixian Tang ${ }^{*(1)}$, Chao wang ${ }^{(1)}$, Hong Zhang ${ }^{(1)}$ \\ ${ }^{(1)}$ Key Laboratory of Digital Earth Science, Institute of Remote Sensing and Digital Earth, CAS, Beijing, China, 100094, \\ Email: yxtang@ceode.ac.cn \\ ${ }^{(2)}$ University of Chinese Academy of Sciences, Beijing, China, 100049, Email:zhangzj01@ radi.ac.cn
}

\begin{abstract}
A modified time-series InSAR technique combing Persistent Scatterers and Distributed scatterers is applied to obtain the deformation information at coal mining areas in Huainan city. In order to select DSs, a selecting strategy combining both classified information and statistical characteristics is used. To control error propagation and improve computational efficiency, a region growing-based strategy is applied to extract the deformation rate at DSs. A series of Radarsat-2 $\mathrm{HH}$ polarization images collected in Huainan over a period of approximately two years are processed to verify the effectiveness of the proposed method. The experimental results show that zones with obvious subsidence are detected in coal mining areas with a maximum deformation rate larger than 10.7 $\mathrm{cm} /$ year during the observation period.
\end{abstract}

\section{INTRODUCTION}

Underground coal mining often causes persistent land subsidence, which would lead to huge threats to infrastructure and safety in the coal area $[1,2]$. Traditional levelling and GPS methods are able to produce reliable measurement of ground subsidence [3]. However, these field surveys are time-consuming and cannot provide deformation map with high spatial sampling density.

Synthetic aperture radar (SAR) interferometry (InSAR) is a powerful technology, which can obtain high precise elevation and surface deformation information along line of sight (LOS) using phase information from different SAR images. However, conventional InSAR usually suffers from temporal, geometrical and atmospheric decorrelation. In order to overcome these limitations, several advanced techniques have been proposed, such as Persistent Scatterer Interferometry (PSI) [4, 5] and Small baseline Subset Algorithm (SBAS) [6]. In recent years, Distributed Scatterers Interferometry (DSI) [7, 8] has been proposed to monitor the surface deformation with high spatial density of measurement points. The main drawback of DSI is the computational efficiency $[7,8]$ in large scale suburb areas.

This paper presents an approach to obtain ground deformation using time-series InSAR technique combining PSs and DSs. PSs are selected based on their coherence stability in the stack of interferograms. In order to identify DSs, a selecting strategy combining both classified information and statistical characteristics is used $[9,10]$. To control the error propagation and improve the computational efficiency, deformation rate and DEM error on PSs are firstly retrieved using conventional PSI. Then a region grown-based strategy is applied to extract deformation rate on the useful DSs. A series of Radarsat-2 HH polarization images collected in Huainan are processed to verify the effectiveness of the proposed method. A dense ground surface deformation map is obtained. The experimental results show that the potential application of the proposed method.

\section{METHOD}

The block diagram of the proposed method is shown in Fig. 1. A selecting strategy combining both classified information and statistical characteristics is used to select DSs. Then the deformation parameters of PSs are retrieved using conventional PSI. Finally a region growing strategy is applied to extract deformation rates of DSs. In this study, we focus on two steps: selection of DSs and parameter estimation of DSs through a region growing method, which will be presented in this section.

\subsection{Selection of DSs}

DSs can be identified from the combination of classification analysis and mathematical statistics method. The former is to identify the specific classes for DSs, such as bare soil, ground and the sparse vegetation areas. The latter is to refine the candidates.

A DS object covers several pixels in high-resolution images, and these pixels are statistical homogeneous pixels (SHP) sharing the same behaviour corresponding to the suburban and soil area. However, water and forest area in the image are also homogeneous pixels, but not belonging to DSs. A prior classification map of the region can be of great help for DSs selection. In this paper, the classification map is generated by utilizing mean amplitude map of the SAR images to identify potential DSs classes.

For the collected candidates, an adaptive sample 
selection strategy is applied to refine them. Varies statistical tests have been proposed in recent years to identify homogenous pixels. The Anderson-Darling (AD) test has been proven to have better performance than Kolmogorov-Smirnov (KS) test [11]. Thus, AD test is adopted in this paper. For each candidate in the image, its statistical homogeneous pixels are identified, which can be applied to adaptive filtering as well as estimation of complex coherence value. And then, those DS pixels are selected for further processing, which possess an average coherence greater than a certain threshold and have a minimum number of SHP.

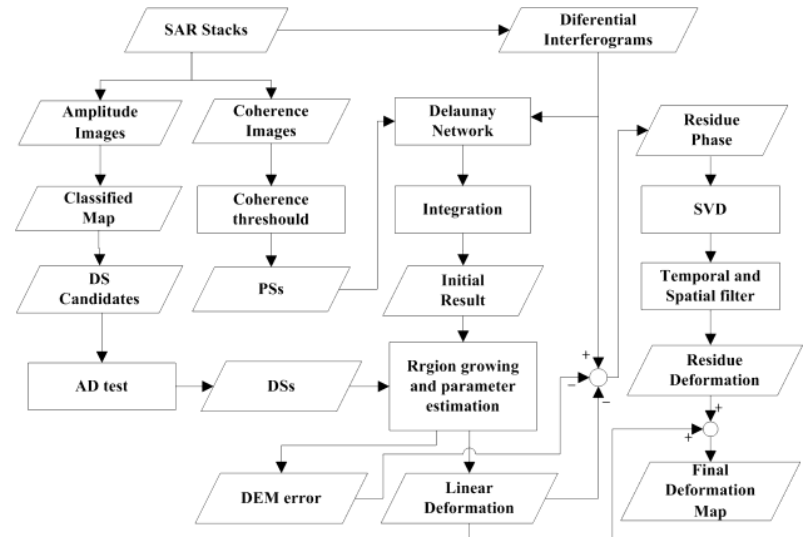

Figure. 1 Block diagram of the proposed method.

\subsection{Estimation Differential Deformation rate and DEM error between two points}

Generally, the phase difference between two neighbouring pixels, $x$ and $y$, on each edge of network in the kth interferogram can be expressed as the following model:

$$
\Delta \varphi_{\text {model }}^{k}=\frac{4 \pi}{\lambda} \cdot T^{k} \cdot \Delta v_{(x, y)}+\frac{4 \pi}{\lambda R \sin \theta} \cdot B_{\perp}^{k} \cdot \Delta \varepsilon_{(x, y)}
$$

Where $T^{k}, B_{\perp}^{k}, \mathrm{R}$ and $\theta$ denote time baseline, normal baseline, slant range, and incidence angle, respectively;

is the wavelength and, are the deformation rate variation and the elevation error variation of two neighbouring pixels.

The differential height error and deformation velocity are generated by maximizing the absolute value of $\xi$,

$$
\xi=\left|\frac{1}{N} \sum_{k=1}^{N} \exp \left[j \cdot\left(\Delta \phi_{\text {phase }}^{k}-\Delta \phi_{\text {model }}^{k}\right)\right]\right|
$$

Where $\mathrm{N}$ is the number of interferograms, $\Delta \phi_{\text {phase }}^{k}$ is the phase difference of the neighbouring two points in the $\mathrm{k}^{\text {th }}$ interferogram. The maximum of the absolute value of (2) is called temporal coherence.

\subsection{Parameters estimation on PSs and DSs}

In order to control the error propagation, deformation rate and topographic error on PSs are firstly retrieved using conventional PSI. After Delaunay network is constructed on PSs, the differential deformation rate and DEM error on each edge of the network are estimated using the method described in section 2.2. Once the estimation of deformation parameter of all edges on PSs has been done, the deformation velocity and DEM error of all the validate points can be obtained through integration step. After getting the linear deformation rate of PSs, the PSs with good quality are preserved and added into the processed target (PT) set. The valid deformation results from PS set are used as the reference data for parameters estimation of DS set.

Because of the large number of the DSs, it will be computationally expensive and difficult to retrieve deformation parameters if constructing Delaunay triangulation on DSs simultaneously. Therefore, a method using region growing strategy is applied to extract deformation rate and DEM error of DS set. Starting from a reference DS, all the DSs can be visited through a region growing method. For each DS, referring it as unprocessed target (UPT), neighbouring processed targets (PTs) from the PT set are identified using a selecting window (see Fig. 2). Then each of the PTs is linked with UPT and $\Delta v, \Delta \varepsilon$ of the links are estimated using the method described in section 2.2. Only the link with temporal coherence large than a given threshold is viewed as validated link. If there is no validated links between UPT and PTs, the UPT is viewed as an invalid point. Otherwise, the LOS subsidence rate and elevation error of the UPT are obtained using (3) and (4) and the UPT is added into PT set:

$$
\begin{aligned}
\mathrm{V}_{C} & =\frac{\sum_{i=1}^{\mathrm{M}} \xi_{i} \cdot\left(V_{i}+\Delta v_{i}\right)}{\sum_{i=1}^{M} \xi_{i}} \\
\varepsilon_{C} & =\frac{\sum_{i=1}^{\mathrm{M}} \xi_{i} \cdot\left(\varepsilon_{i}+\Delta \varepsilon_{i}\right)}{\sum_{i=1}^{M} \xi_{i}}
\end{aligned}
$$

Where $V_{C}, \varepsilon_{C}$ are the estimated deformation rate and DEM error; $\mathrm{M}$ is the number of the validated links; $v_{i}$, $\varepsilon_{i}$ are the deformation rate and DEM error of the $\mathrm{i}^{\text {th }} \mathrm{PT}$, respectively; $\Delta v_{i}, \Delta \varepsilon_{i}, \xi_{i}$ denote the deformation rate, DEM error, and temporal coherence of the link between UPT and the ith PT, respectively. In the same way, all the UPT are analysed to retrieve the deformation rate and DEM error.

Once the linear deformation rate of all the points have been obtained, the nonlinear deformation components can be extracted using spatial and temporal filtering at both PSs and DSs. 


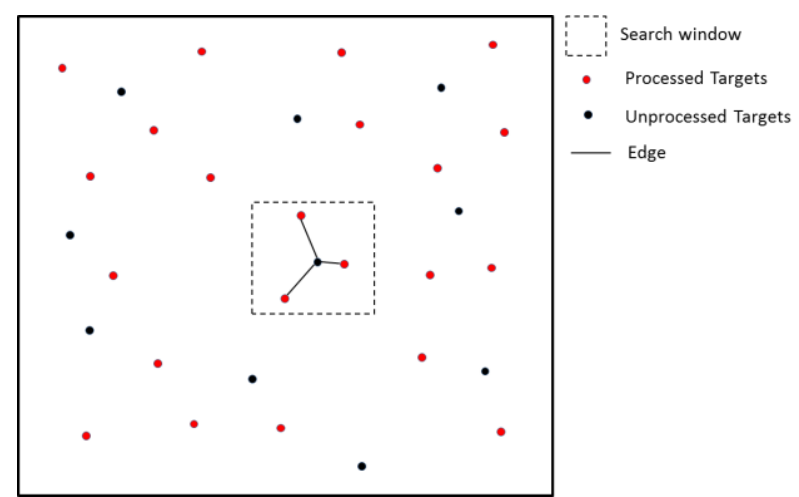

Figure. 2 Sketch of identifying processed targets

\section{STUDY AREA AND DATA}

Huainan, located in the Yangtze River Delta hinterland central of Anhui province, is rich in coal resource, accounted for $19 \%$ of the national vision of stocks of coal stock. About 14 pairs of key state-owned coal mines are located in Huainan City. However, this anthropogenic activity has caused series land subsidence, which result in many series of environmental problems and damages. As shown in Fig. 3, the central part of Huainan is selected as the study area, in which there are five coal mining areas, Dingji, Guqiao, Zhangjiaji, Xinji I and Xinji II. In the coal mining areas, there are several collapsed lakes resulting from coal mining.

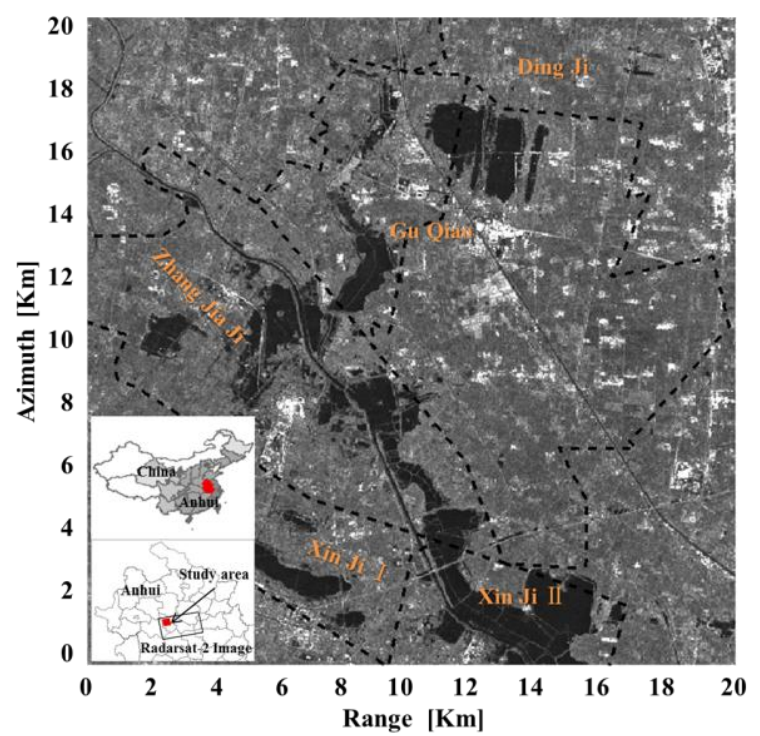

Figure. 3 Location map of the study site and amplitude image. Five coal areas are marked. The two insets show the location of Anhui province in China and the coverage of a Radarsat-2 image

In order to monitor the subsidence of the coal mining area in Huainan city, 20 Radarsat-2 ascending images acquired from September 2012 to May 2014 with a look angle of 36.6 degrees and ' $\mathrm{HH}$ ' polarization have been used. The SAR amplitude images (about $20 \times 20 \mathrm{~km}^{2}$ ) averaged from all the images for the study area is shown in Fig.3. The pixel spacing of range and azimuth is 2.66 $\mathrm{m}$ and $2.49 \mathrm{~m}$, respectively. 97 interferometric pairs are combined to generate interferograms with perpendicular baseline less than $200 \mathrm{~m}$ and temporal baseline less than 200 days.

\section{EXPERIMENTAL RESULT}

After generating the average coherence map, point with coherence value large than 0.65 is selected as PS. 70939PSs are identified as shown in Fig. 4(A). The unsupervised IsoData method is used to generate the classification map based on the mean amplitude image. By removing the non-DSs classes, the DS candidates are obtained. Subsequently, AD test with a window of $11 \times 11$ is applied to select the DSs which have an average coherence larger than 0.45 and the number of SHP larger than 70. 318,561 DSs have been selected by the proposed selection strategy (approximately 856 DSs $/ \mathrm{km}^{2}$ ), which is shown in Fig. 4(B). It is shown that the density of selected DSs is much higher than that of PSs in rural area.
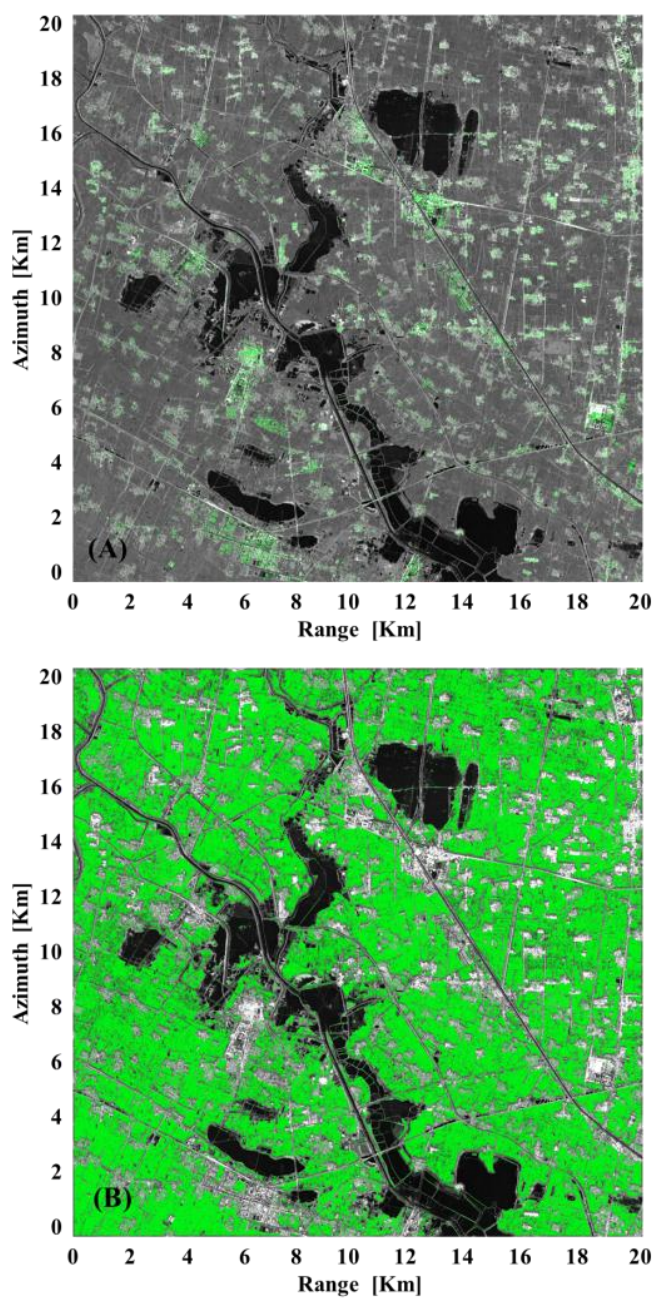

Figure.4 (A) selected PSs; (B) selected DSs

After generating the deformation information at PSs, the 
deformation rate and DEM error at DSs are retrieved with the proposed method in this paper. Experimental result at PSs is shown in Fig. 5(A). And Fig. 5 (B) gives the estimated deformation rate at both PSs and DSs. because of the wide distribution of DSs, The spatial density of measurement points is high over the whole image. The average subsidence velocity of the study area is about $53.3 \mathrm{~mm}$ /year. It is shown that four subsidence areas are marked by red rectangle line with a peak subsidence rate of 107 mm/year. In Fig. 5 (B), five subsidence areas (marked with black boxes) are detected in the study site. Those subsidence areas are located in the central of the coal mining area, which indicates that coal mining contribute to the ground movement.
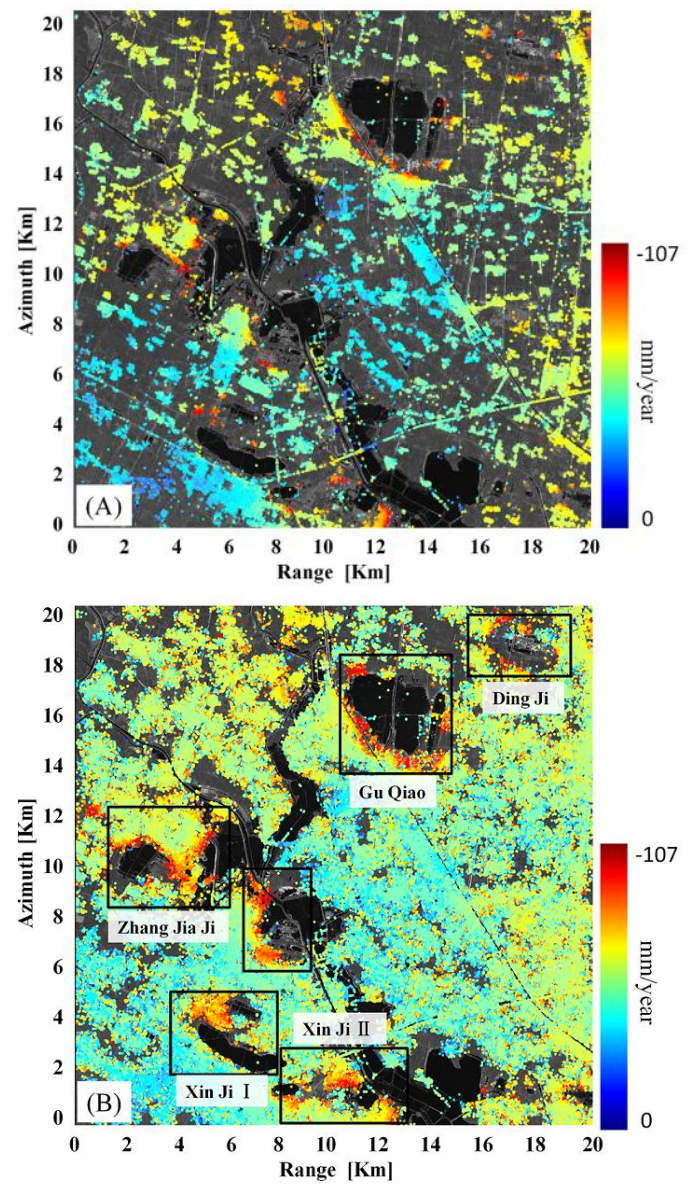

Figure. 5 Estimated subsidence velocity map at (A) only PSs and (B) both PSs and DSs. Five subsidence centres are detected in this area marked by a black rectangular line.

\section{CONCLUSION}

This paper has presented an approach to extract ground movement in coal mining area using time-series InSAR on PSs and DSs, thus resulting in more detail of the subsidence measurements. DSs can be efficiently identified using information and statistical property. A region growing strategy has been applied in the processing of DSs, which may make parameter estimation easier and more efficiency. A dense ground surface deformation map of the study area is obtained, by employing 20 Radarsat-2 images over Huainan city, China, which could make it easy to identify the process responsible for land subsidence. The experimental results show that subsidence zones are detected in coal mining areas with a maximum deformation rate of 10.7 $\mathrm{cm} /$ year. Further work will focus on modelling the process of the coal mining in the parameter estimation step.

\section{ACKNOWLEDGEMENTS}

This work was supported by the National Natural Science Foundation of China under Grants 41271425 and 41331176. The authors would like to thank Canadian Space Agency for Radarsat-2 data.

\section{REFERENCE}

[1] H. C. Jung, S.-W. Kim, H.-S. Jung, K. D. Min, and J.-S. Won, "Satellite observation of coal mining subsidence by persistent scatterer analysis," Eng. Geol., vol. 92, pp. 1-13, 2007.

[2] J. Baek, S.-W. Kim, H.-J. Park, H.-S. Jung, K.-D. Kim, and J. W. Kim, "Analysis of ground subsidence in coal mining area using SAR interferometry," Geosci. J., vol. 12, pp. 277284, 2008

[3] A. Demoulin, J. Campbell, A. D. Wulf, A. Muls, R. Arnould, B. Görres, et al., "GPS monitoring of vertical ground motion in northern Ardenne-Eifel: five campaigns (19992003) of the HARD project," Int. J. Earth. Sci., vol. 94, pp. 515-524, 2005.

[4] A. Ferretti, C. Prati, and F. Rocca, "Permanent scatterers in SAR interferometry," IEEE Trans. Geosci. Remote Sens., vol. 39, pp. 8-20, 2001.

[5] A. Hooper, H. Zebker, P. Segall, and B.Kampes, "A new method for measuring deformation on volcanoes and other natural terrains using InSAR persistent scatterers," Geophys. Res.Lett., vol. 31, 2004.

[6] P. Berardino, G. Fornaro, R. Lanari, and E.Sansosti, "A new algorithm for surface deformation monitoring based on small baseline differential SAR interferograms," IEEE Trans. Geosci. Remote Sens., vol. 40, pp. 2375-2383, 2002.

[7] A. Ferretti, A. Fumagalli, F. Novali, C. Prati, F. Rocca, and A. Rucci, "A new algorithm for pro-cessing interferometric data-stacks: Squeesar," IEEE Trans. Geosci. Remote Sens., vol. 49, pp. 3460-3470, 2011.

[8] K. Goel and N. Adam, "An advanced algorithm for deformation estimation in non-urban areas," ISPRS $J$. Photogram. Remote Sens., vol. 73, pp. 100-110, 2012.

[9] Z. Zhang, C. Wang, Y. Tang, Q. Fu, and H. Zhang, "Subsidence monitoring in coal area using time-series InSAR combining persistent scatterers and distributed scatterers," Int. J. Appl. Earth. Obs., vol. 39, pp. 49-55, 2015.

[10] Z. Zhang, C. Wang, Y. Tang, H. Zhang, and Q. Fu, "Analysis of ground subsidence at a coal mining area in Huainan using time-series InSAR," Int. J. Remote. Sens .(in review)

[11] A. Parizzi, and R. Brcic, "Adaptive InSAR stack multilooking exploiting amplitude statistics: a comparison between different techniques and practical results," IEEE Trans. Geosci. Remote Sens., vol. 8, pp.441-445, 2011. 